\title{
Arme Tiere: die Option für die Armen als möglicher Anschlusspunkt einer christlichen Tierethik
}

\author{
Julia Blanc
}

\section{Ruf nach christlicher Tierethik und theologischer Beachtung von Tieren ist vernehmbar ${ }^{1}$} \begin{abstract}
„Also ich habe, ich habe halt Haustiere gehabt, habe mich für Naturethik und generell für Philosophie interessiert. Dadurch habe ich gemerkt - das ist meine persönliche Überzeugung - dass wir in Deutschland einen Zustand haben, über den wir in 30 Jahren traurig sein werden. Sage ich jetzt einfach mal. Das ist meine persönliche Überzeugung. In 30, 40 Jahren werden wir darüber sprechen, wie wir Tiere gehalten haben, wie wir mit der Umwelt umgegangen sind und werden sagen, Wie kann man nur so verantwortungslos umgehen mit der Schöpfung, mit den Lebewesen, mit der Erde die uns gegeben ist für einen bestimmten Zeitraum?
\end{abstract}

Das kommt aus meiner persönlichen Biografie.

Ich merke aber, dass wenn ich mit Pastoren, also mit [theologisch] gebildeten Leuten in unseren Kreisen spreche, dass die sagen ,Ja wir haben da definitiv ein Defizit““ (Samuel, Interview 15, 02.10.2018²).

So ein evangelikaler Pastor, der im Rahmen des Forschungsprojekts Urban Green Religions ${ }^{3}$ interviewt worden ist.

Ähnliche Antworten ergaben sich auch bei Gesprächen mit vorwiegend anderen christlichen Akteuren: Katholische Verantwortliche für die Orga-

1 Die qualitativen Daten, welche diesem Beitrag zu Grunde liegen, wurden im Rahmen des SNF-geförderten Forschungsprojekts Urban Green Religions an der Universität Basel erhoben.

2 Name durch Autorin anonymisiert. Satzzeichenfehler im Original.

3 Das durch den Schweizerischen Nationalfonds (SNF) finanzierte, an der Universität Basel beheimatete Forschungsprojekt erhob seit April 2018 über 70 qualitative Leitfadeninterviews mit Vertreter*innen von Kirchen, religiösen Gemeinschaften, spirituellen Zentren, Stadtverwaltungen und Akteuren aus Wirtschaft und Politik in den beiden Benchmark-Cities in Süddeutschland und der Innerschweiz. Diese wurden mit Hilfe der Datenanalysesoftware MAXQDAs ausgewertet. 
nisation und Durchführung von Jugendlagern verwiesen auf die bewusst fleischarme, dafür biologisch und regional produzierte Ernährung, welche auch durch finanzielle Anreize gefördert wurde. Ein protestantischer Pfarrer sprach sich für den Erhalt und weiteren Ausbau von Streuobstwiesen als natürliches Habitat von Insekten, Vögeln und anderen Tieren aus. Hierfür trägt er beispielsweise Sorge, indem er ausschließlich die im Ort produzierten Säfte beim Pfarrfest anbietet. Von Seiten der Deutschen Bischofskonferenz (DBK) wie auch der Evangelischen Kirche in Deutschland (EKD und ihrer vor Ort beheimateten Gliedkirche wurde auf mannigfaltige Veröffentlichungen zum Thema Tierwohl und Mitgeschöpflichkeit meist im Rahmen von in landwirtschaftlichen Belangen veröffentlichten Schreiben - verwiesen. Besonders der von der EKD veröffentlichte Text Nutztier und Mitgeschöpf! Tierwohl, Ernährungsethik und Nachhaltigkeit aus evangelischer Sicht ${ }^{4}$ greift die tierethische Diskussion auf und müht sich um eine Verankerung in der Theologie.

Trotzdem oder gerade wegen des „irgendwie“ bestehenden Interesses oder auch der Offenheit für theologische Begründungen bezüglich einer vermehrten Achtung vor nicht-menschlichem Leben sind Tiere bislang im theologischen Diskurs insbesondere als Individuen, als Gegenüber, nur sehr marginal vertreten (Julia Blanc 2011). Die zentrale Interrogation des Textes ist es deshalb, ob sich in der Option für die Armen ein Anschlusspunkt für eine - dann logischerweise christliche - Tierethik, besser Tiertheologie, findet. Die Unterscheidung tut deshalb not, weil sich die immer noch sehr geringe Auseinandersetzung mit Tieren in der Theologie, der theologischen Ethik und der theologisch geprägten Tierphilosophie hauptsächlich auf moralisch-ethische Argumente stützt (klassisch Andrew Linzey 2009 a und 2009 b; im deutschen Sprachraum: Jochen Ostheimer 2018; Kurt Remele 2016; Markus Vogt 2019). Manch andere gehen noch den Weg über den klassischen Ansatz des Artenschutzes (Markus Vogt 2018; Clemens Wustmans 2015). Michael Rosenberger (2015) bezieht theologische Argumente in seinen auf ein Gegenüber konzentrierten Ansatz mit

4 EKD-Text 133 „Nutztier und Mitgeschöpf! Tierwohl, Ernährungsethik und Nachhaltigkeit aus evangelischer Sicht“ (https://www.ekd.de/ekd_de/ds_doc/ekd_texte_ 133_2019.pdf; abgerufen am 05.05.2020) verweist unter anderem auf verschiedene Bibelstellen, die Mensch und Tier nebeneinander stellen: Menschen und Tiere sind Geschöpfe Gottes, sie tragen beide den gleichen von Gott geschenkten Lebensatem (Ps 104,10-18). Menschen und Tiere stehen beide unter den Segens- und Schutzverheißungen Gottes (Gen 9,16). Die biblischen Friedensvisionen für eine neue Welt schließen die Tiere ausdrücklich mit ein (Jes 65,1-25). 
ein, fokussiert dabei aber, Linzey folgend, auf die Mitgeschöpflichkeit ${ }^{5}$ der nicht-menschlichen Tiere. Den Tieren oder dem einzelnen Tier zugesprochene Würde stellt den zentralen Ansatz bei Martin M. Lintner (2017) dar, der dabei in Bezug auf Fleischkonsum bzw. Tierproduktverzicht ähnlich wie Eberhard Schockenhoff (22013) argumentiert. Simone Horstmann u. a. und wiederum gehen einen "theologischeren“ Weg, bei dem sie insbesondere biblische und frühchristliche Zeugnisse von Tierwertschätzung zusammentragen, einordnen und bewerten (Simone Horstmann et al. 2018; Simone Horstmann 2020).

Als Antwort auf das eingangs vom evangelikalen Pastor formulierte Desiderat soll in den folgenden Seiten ein möglicher Ansatz angedacht und vorgestellt werden.

Dafür wird erst die lehramtliche Meinung, insbesondere die in vielen Ansätzen „neue“ Position von Papst Franziskus näher beleuchtet. Danach wird sie in einen Zusammenhang mit der dafür grundlegenden Lehrmeinung der katholischen Kirche zur Situation nicht-menschlichen Lebens abgeglichen. Hierauf erfolgt eine vertiefte Auseinandersetzung mit der Option für die Armen, wie sie von Ignacio Ellacuria (1996), im deutschen Sprachraum auch durch Giancarlo Collet (1992) in den letzten Jahrzehnten geprägt worden ist. Die Zusammenschau dieser beiden Blöcke sollte es ermöglichen, die Potentiale und ihre Grenzen einer solchen „Ausweitung“ der Option für die Armen großflächig einzuordnen.

\section{Kirchliches Lehramt}

Mit der Wahl Jorge Mario Bergoglios 2013 zum Papst und dessen programmatischer Namenswahl als Franziskus wurde dessen für sein Pontifikat tragend werdende Hinwendung zu den am Rande Lebenden bereits vorweggenommen. Dabei wird von vielen seine nicht-europäische Herkunft als Grund für sein „untypisches“ Verhalten angeführt. Diese Offenheit für unkonventionelle Ansätze, die nicht nur dem klassisch Erwarteten bei päpstlichen Äußerungen nur sehr bedingt folgt, sondern dabei auch noch tradierte Vorstellungen zum Primat des Menschen nachhaltig in Frage stellt, verdeutlicht seine neue Herangehensweise.

5 Tierschutz-ethischer Ansatz, der auf die gemeinsame Geschöpflichkeit der Natur fokussiert, also das Wissen um das gemeinsame Kommen und Gewolltsein von Gott betont. 
Ebenfalls durch seine Herkunft und seinen theologischen Hintergrund bedingt ist fraglos seine größere Offenheit gegenüber zumeist aus Südamerika stammenden Ansätzen der Option für die Armen, wie er sie an verschiedensten Stellen rezipiert; sprichwörtlich wurde diesbezüglich bereits sein Wunsch nach einer „armen Kirche für die Armen“, so Papst Franziskus bei seiner ersten Audienz für Medienvertreter am 16. März 2013. Diesem Ruf nach einer Rückbesinnung auf urchristliche Wurzeln wurde innerhalb der katholischen Kirche unter anderem mit seiner Hervorhebung und Revalorisierung des Katakombenpakts pünktlich zu dessen fünfzigstem Jahrestag Achtung gezollt. Auch seine die Flexibilität bzw. Reformbereitschaft seiner Amtsvorgänger bei weitem übersteigende Bereitschaft, sich mit den unterschiedlichen Herausforderungen der Gegenwart auseinanderzusetzen und neuen Ideen nicht grundlegend abgeneigt gegenüberzustehen, lässt auf eine weitergehende Überarbeitung der kirchlichen Position vis-à-vis nicht-menschlicher Mitgeschöpfe hoffen. Diesbezüglich sei auf den innerkirchlich-lehramtlichen Fortschritt im Bereich der Umweltethik verwiesen, die zwar keinesfalls mit der Diskussion um eine adäquate Behandlung nicht-menschlichen Lebens gleichgesetzt verstanden werden soll, deren Entwicklung in den letzten 50 Jahren aber durchaus Anknüpfungspunkte, Parallelen und Inspiration bietet: während Paul VI. zwar mit seiner Entwicklungsenzyklika Populorum progressio (1967) einerseits heute kaum mehr nachvollziehbare Elogien auf den Fortschritt und damit auf den Menschen im Fortschritt verfasste, näherte er und damit die katholische Kirche sich in ihrer fast 2000-jährigen Geschichte erstmals dem Themenkomplex „Umwelt“. Da sowohl der Bericht des Club of Rome mit seinem Hinweis auf die Grenzen des Wachstums (Donella Meadows et al. 1972) als auch das zum Teil darauf aufbauende Bewusstsein der Menschheit für Erdölknappheit und Artensterben nicht gänzlich vom Vatikan und den Päpsten ignoriert wurde (oder ignoriert werden konnte?), sah sich Papst Johannes Paul II. gezwungen, auch die kirchliche Position zu überdenken bzw. diese in einen zeitlich bedingten Kontext zu setzen. Dies geschah mit der Einführung bzw. Übernahme des Konzepts der „HumanÖkologie" durch das päpstliche Lehramt. ${ }^{6}$ Durch diesen multiperspektivischen Ansatz, der über Jahrzehnte hinweg stark von Soziologie und Biologie, aber auch von Anthropologie, Ethnologie, Geographie, Psychologie

6 Namentlich die erste Erwähnung erfolgt 1991 in Centesimus annus, Nr. 38. Das Konzept lässt sich aber bereits in Redemptor hominis, Nrn. 8, 14, 15, 16, Sollicitudo rei socialis, Nrn. 26, 29, 30, 34 und der Botschaft zum Weltfriedenstag 1990 mit dem Titel „Friede mit Gott, dem Schöpfer - Friede mit der ganzen Schöpfung“ nachweisen (Markus Vogt 2013). 
und Medizin geprägt wurde, gelang es, weiterhin den Primat des Menschen aufrecht zu erhalten und gleichzeitig den Aspekt der aggressiven Dominanz zu mindern (Markus Vogt 2013). Eine weiterführende Rezeption im kirchlichen lokalen Leben jedoch scheiterte besonders im deutschsprachigen Raum. Daran nicht unschuldig dürfte die „schillernde Vielschichtigkeit und Heterogenität“ (Markus Vogt 2013, 251) des Begriffs sein. Mit Letzterem ist die einseitige Konzentration auf den Vorteil des Menschen, wie sie im deutschsprachigen Raum vor allem von Carl Amery (1972) angeprangert wurde, gemeint. Diese als dominium-terrae-Vorwurf bekannt gewordene Position macht sich an dem Verb kabasch, genauer an dessen einseitiger Übersetzung in Gen 1,28 fest. Denn entgegen der heute gängigen Interpretation, die den Fokus auf „dienstbar/urbar machen“ legt, wurde es lange Zeit ausschließlich als „unterwerfen“ verstanden. Dies führte zu einer übermäßigen Fokussierung auf den Menschen als Krone der Schöpfung.

Hierdurch wurde es nur sehr bedingt geschafft, die international durch Lynn White (1967) erfolgte Brandmarkung des Christentums bzw. der katholischen Kirche als Ursprung allen ökologischen Übels auszuradieren. Der von Letzterem formulierte Vorwurf resultiert aus der Technikbegeisterung mittelalterlicher Mönche, ohne die eine industrielle Revolution, wie sie Europa und in Folge dessen auch die USA im „langen“ 19. Jahrhundert erlebt haben, nicht denkbar gewesen wäre, so das zentrale Argument Whites. Erst die Überzeugung der Mönche, bei der Anwendung neuester wissenschaftlicher Erkenntnisse „gottgefällig“ zu handeln, führte zur rasanten Verbreitung unterschiedlichster Hilfsmittel zur „Unterwerfung“ der Erde und letztlich zur „aktuellen ökologischen Krise“, so der Autor weiter. Diesem Vorwurf wurde in den letzten Jahren und Jahrzehnten aus historischer, biblischer und systematischer Sicht (die deutschsprachige Kritik setzt u. a. bei Hans J. Münk 1987 und Simone Rappel 1996 ein) nachdrücklich widersprochen. Gleichwohl kann und muss der Text auch als Fanal für das gesamte Interesse kirchlicher und religiöser Akteure, aber auch der gesamten religionswissenschaftlichen Beschäftigung mit dem Umweltbereich verstanden werden: Bron Taylor, der Gründer der ISSRN (International Society for the Study of Religion and Nature) ${ }^{7}$, wie auch Mary Eve-

7 Vgl. www.religionandnature.com (abgerufen am 04.05.2020), mit einem Fokus auf „The Encyclopedia of Religion and Nature“ aus dem Jahr 2006. 
lyn Tucker und John Grim ${ }^{8}$ haben sich auf Lynn Whites Beschuldigungen des Christentums bei der Begründung eines vollkommen neuen Forschungsfeldes bezogen bzw. reagieren mit ihrer Arbeit auf seine Vorwürfe.

Kritisch kann, muss und sollte an dieser Stelle fraglos auf die „kurze“ theologische, vor allem katholische Tradition seit dem 2. Vatikanischen Konzil verwiesen werden, deren Ergebnisse in der Pastoralkonstitution Gaudium et spes und letztlich auch im Katechismus der Katholischen Kirche (KKK) Niederschlag gefunden haben. Diese stellt potentiell eine Verschlechterung des tierischen Status Quo, wie er sich aus der Bibel ableiten lässt, dar. Camosy und Kopp verweisen nachdrücklich auf die Tatsache, dass die unterschiedlichen Tiere am vierten und am fünften Schöpfungstag - also vor dem Menschen - geschaffen wurden und auch ohne diesen bereits "gut" waren (Charles Camosy/Susan Kopp 2014, auch Simone Horstmann et al. 2018, 50). Schwierig ist in diesem Zusammenhang fraglos KKK, Nr. 356, in dem explizit auf GS 24,3 Bezug genommen wird:

„Von allen sichtbaren Geschöpfen ist einzig der Mensch ,fähig, seinen Schöpfer zu erkennen und zu lieben' (GS 12,3); er ist ,auf Erden das einzige Geschöpf ... das Gott um seiner selbst willen gewollt hat' (GS 24,3); er allein ist berufen, in Erkenntnis und Liebe am Leben Gottes teilzuhaben. Auf dieses Ziel hin ist er geschaffen worden, und das ist der Hauptgrund für seine Würde [...].“

Dem gegenüber sprechen KKK, Nrn. 2415-2418, eine zumindest auch anders deutbare Sprache. So ist zwar das Nutzen von Tieren innerhalb von „vernünftigen Grenzen sittlich zulässig“ (Nr. 2417), ihr Quälen wird aber verworfen (Nr. 2418). Franz von Assisi und Philip Neri werden als Vorbilder im rücksichtsvollen Umgang mit Tieren explizit erwähnt (Nr. 2416).

Diese Bandbreite (und noch mehr) der dargestellten Bewertung nichtmenschlicher Tiere im theologischen Kontext spiegelt sich (auch) im Werk von Papst Franziskus wider.

8 Vgl. https://fore.yale.edu/Event-Listings/Religions-World-and-Ecology-ConferenceSeries/Religions-World-and-Ecology-Archive (abgerufen am 04.05.2020), wo auf die Konferenzen, welche zwischen 1996-1998 stattgefunden haben, und die daraus entstandene und von ihnen herausgegebene Buchreihe (Religions of the World and Ecology, 1997-2004) verwiesen wird. 


\subsection{Evangelii gaudium}

Das Apostolische Schreiben Evangelii gaudium (Franziskus 2013) ist nicht die erste Veröffentlichung von Franziskus, aber zumindest die erste, welche ausschließlich seine Handschrift trägt. Programmatisch und wegweisend wie seine Namenswahl lesen sich aus heutiger Sicht die Schwerpunkte: Auswüchse der globalen Wirtschaftsordnung werden angeprangert und erste Linien für eine zeitgemäße Verkündigung der christlichen Botschaft sowie für eine Reform der Kirchenstrukturen skizziert. Wenn auch ökologische Themen immer wieder anklingen, steht der Mensch noch immer im Zentrum der Reflexion, insbesondere jene Menschen am Rande der Gesellschaft. In Evangelii gaudium, Nr. 53, formuliert Franziskus:

„Der Mensch an sich wird wie ein Konsumgut betrachtet, das man gebrauchen und dann wegwerfen kann. Wir haben die, Wegwerfkultur ${ }^{6}$ eingeführt, die sogar gefördert wird. Es geht nicht mehr einfach nur um das Phänomen der Ausbeutung und der Unterdrückung, sondern um etwas Neues: Mit der Ausschließung ist die Zugehörigkeit zu der Gesellschaft, in der man lebt, an ihrer Wurzel getroffen, denn durch sie befindet man sich nicht in der Unterschicht, am Rand oder gehört zu den Machtlosen, sondern man steht draußen. Die Ausgeschlossenen sind nicht ,Ausgebeutete', sondern Müll, ,Abfall‘““

Diese Interpretation der Armen als „Ausgeschlossene“ der Gesellschaft ist nicht neu, sondern wurde beispielsweise durch die Idee der „Nichtperson“ (Gustavo Gutiérrez ${ }^{10} 1992$ ) oder der Illegalen9 (Milton Schwantes 2002) vorweggenommen. Bislang hatte sich die Kirche bzw. das Lehramt immer um eine dezidierte Unterscheidung zwischen Menschen und Nicht-Menschen bemüht. Nun bietet dieses Aufbrechen der klassischen Kategorien auch Chancen, vor allem für den bislang ,vernachlässigten“ Teil des nichtmenschlichen Lebens. Dabei möchte vorliegender Beitrag keineswegs menschliches und nicht-menschliches Leben gleichsetzen. Vielmehr soll der Blickwinkel, unter dem nicht-menschliches Leben betrachtet wird, verschoben werden. Hierzu könnte eine veränderte theologische Wahrnehmung von Tieren beitragen.

9 ... da sie illegal wohnen, arbeiten, leben etc. 


\subsection{Laudato si'}

Ebenfalls diesem nicht-menschlichen Leben widmet Franziskus 2015 seine zweite Enzyklika Laudato si' (LS), der als „Umweltenzyklika“ auch und vor allem von nicht-theologischer Seite größte Bedeutung zugemessen wird (Mariano Pasquale Barbato 2018; Katharina Glaab et al. 2019). ${ }^{10}$ Die EKD hat sich dieser positiven Beurteilung explizit in Bezug auf Tiere angeschlossen (EKD 2019 u. a. 7, 17 und 121).

Die nicht-katholische Würdigung ist besonders in Hinblick auf die theologische und sozialethische wie auch pastorale Bewertung der Umwelt als eigener Kultursachbereich bedeutend; letztlich kann und muss eine Parallele zur Bedeutung von Rerum novarum (Leo XIII. 1891) als Initialdokument römischen Interesses in und an der Arbeiterfrage erkannt werden (Julia Blanc 2017). In der Literatur wird dieser Wandel auch als sogenannter Francis Effect (Edward Maibach et al. 2015) bezeichnet. Maibach et al. beziehen sich damit auf die in der greening-of-religions-Debatte prominente Meinung, dass Religion, im vorliegenden Fall der katholischen Kirche, von außen Potential im Umgang mit ökologischen Herausforderungen zugestanden wird, das sie in sich selbst nicht zwingend vermutet und fördert.

Was aber unter „Umwelt“ subsummiert wird, macht Franziskus schnell deutlich: Bewusst lässt er den rein naturalistischen Rahmen hinter sich und macht den Zusammenhang aktueller Fehlentwicklungen deutlich. „Es gibt nicht zwei Krisen nebeneinander, eine der Umwelt und eine der Gesellschaft, sondern eine einzige und komplexe sozio-ökologische Krise" (LS 139). Dabei ist bedeutsam, dass der Mensch die Natur und in Folge dessen die Natur - bzw. das, was davon übrig ist - auch den Menschen krank macht. Außerdem gibt es für ihn keinen Anlass, „einen despotischen An-

10 Natürlich hätte die Auseinandersetzung - vor allem explizit mit Tieren - noch sehr viel mehr umfassen und ins Detail gehen können, sollen und müssen. Andererseits ist das über nicht-menschliches Leben Gesagte (und die diesem meist entgegengebrachte Achtung) so viel weitergehend, als es vor allem die katholischen Positionsbezüge des vergangenen Jahrhunderts (v. a. KKK und GS) hoffen ließen. Camosy (2015) fasste die Ergebnisse folglich auch als drei Hoffnungsschimmer und drei verstrichene Möglichkeiten knapp und verständlich zusammen. Positiv muss nach ihm die Betonung des inneren Werts auch von Tieren (u. a. in den Nrn. 33, 36, 67, 140), die strikte Zurückweisung von großen landwirtschaftlichen Einheiten (Nr. 129) und die explizite Erwähnung von Tierversuchen bewertet werden. Mängel sieht er in der Nicht-Unterscheidung und -Spezifizierung des Lebens (sind Bakterien, Pilze und Säugetiere wirklich gleich schützenswert?), dem nicht stärker ins Detail Gehen in Bezug auf industrielle Landwirtschaft und die nicht ausgeführte Unterscheidung der Schöpfungsstufen. 
thropozentrismus, der sich nicht um die anderen Geschöpfe kümmert" (ebd., 68), weiter aufrecht zu erhalten und zu verfolgen. Dieser weiche bzw. „gezüchtigte“ (chastened nach Bernard Häring 1981, 180-181, zitiert nach Anatoly Angelo R. Aseneta 2017, 240) Anthropozentrismus zieht zwar immer noch eine deutliche Grenze zu biozentrischen Ansätzen, geht aber weit über die (teilweise) im Katechismus vertretene Qualifizierung nicht-menschlichen Lebens hinaus (Anatoly Angelo R. Aseneta 2017). Nach Kurt Remele (2016) wendet sich diese Formulierung gegen die Ignoranz, mit welcher der Mensch seine nicht-menschlichen Mitgeschöpfe behandelt. Schließlich sei es nach Desmond Tutu „eine Art theologischer Schwachsinn zu glauben, dass Gott die gesamte Welt nur für die Menschen gemacht habe oder dass Gott nur an einer Spezies unter den Millionen Lebewesen, die Gottes gute Erde bevölkern, interessiert sei“, so Remele (2016, 13; er bezieht sich auf Desmond Tutu 2013) weiter. Denn „[w]ährend wir die Dinge in verantwortlicher Weise gebrauchen dürfen, sind wir zugleich aufgerufen zu erkennen, dass die anderen Lebewesen vor Gott einen Eigenwert besitzen“ (LS 69). Dass dies für belebte und nicht-belebte Umwelt gilt, daran besteht kein Zweifel. Folgerichtig formuliert Franziskus weiter unten in seiner Schrift:

„Wenn andererseits das Herz wirklich offen ist für eine universale Gemeinschaft, dann ist nichts und niemand aus dieser Geschwisterlichkeit ausgeschlossen. Folglich ist es auch wahr, dass die Gleichgültigkeit oder die Grausamkeit gegenüber den anderen Geschöpfen dieser Welt sich letztlich immer irgendwie auf die Weise übertragen, wie wir die anderen Menschen behandeln. Das Herz ist nur eines, und die gleiche Erbärmlichkeit, die dazu führt, ein Tier zu misshandeln, zeigt sich unverzüglich auch in der Beziehung zu anderen Menschen. Jegliche Grausamkeit gegenüber irgendeinem Geschöpf ,widerspricht der Würde des Menschen'. Wir können uns nicht als große Liebende betrachten, wenn wir irgendeinen Teil der Wirklichkeit aus unseren Interessen ausschließen“ (ebd., 92; Hervorhebungen durch die Autorin).

Die Zusammenschau der verschiedenen Veröffentlichungen von Franziskus $^{11}$ zur Bewertung nicht-menschlichen Lebens in der heutigen Theologie lassen sich als der Beginn eines Umdenkens lesen. Dabei bilden die ein-

11 Ergänzend sei auf folgende Veröffentlichungen von Papst Franziskus verwiesen, in denen er Tiere positiv erwähnt: Botschaft des Heiligen Vaters Papst Franziskus zur Feier des Weltfriedenstages am 1. Januar 2016, in: http://www.vatican.va/cont ent/francesco/de/messages/peace/documents/papa-francesco_20151208_messaggio -xlix-giornata-mondiale-pace-2016.html (abgerufen am 06.04.2020); Botschaft von 
gangs bereits erwähnten Verallgemeinerungen und zum (Groß)Teil zu undifferenziert ausgefallenen Positionsbezüge (Charles Camosy 2015) eine Angriffsfläche. Auch ein Jahr später bleibt Camosy jedoch seiner Position treu: Die Tatsache, dass Franziskus eine Enzyklika unter anderem dem nicht-menschlichen Leben widmet, wiege die durchaus gravierenden Mängel im Detail allemal auf. Diesem Aspekt kommt vor allem große Bedeutung zu, da ein Rückschritt dahinter als quasi unmöglich ausgeschlossen wird (Charles Camosy 2016). Diese Ambivalenz in der Beurteilung nichtmenschlicher Tiere in Laudato si' sieht auch Anatoly Angelo R. Aseneta (2016; ebenso Martin M. Lintner 2020) und bezieht eine ähnliche Position, wenn er das Gewicht der Valorisierung dieses Lebens über die Behandlung von „Detailfragen“ stellt.

Der Ansatz, „das Tier“ und nicht „die Tiere“ in das Zentrum vorliegender Untersuchung zu stellen, legt es nahe, im nächsten Schritt den Bereich der Bewertung des einzelnen Tieres stärker aus dem Konglomerat rund um Umweltethik und Artenschutz zu befreien. Dieser Zugang sollte mit seiner Konzentration auf „das“ Tier als Gegenüber einem weiterhin (zu) anthropozentrisch ausgerichteten „Tierschutz zum Wohl des Menschen“ einen ergänzenden Aspekt hinzufügen. Vor allem aber zeigen Untersuchungen aus dem Bereich Environment/Nature and Religion, dass religiöse Motivation mehr/andere Menschen zu erreichen weiß als ein rein wissenschaftlicher oder auch ethischer Diskurs (John Grim 2011; Martin Palmer 2013; Maryam Dimaghani 2018). Dieses Potential gilt es nun auch im Sinne der Tiere wie auch der Relevanz religiöser Positionsbezüge in der Öffentlichkeit zu nutzen.

Eine theologische Begründung soll deshalb mit Hilfe der Option für die Armen gesucht werden.

Papst Franziskus zum Welternährungstag 2016 am 14. Oktober 2016, in: http://w ww.vatican.va/content/francesco/de/messages/food/documents/papa-francesco_20 161014_messaggio-giornata-alimentazione.html (abgerufen am 06.04.2020); Nachsynodales apostolisches Schreiben Christus vivit von Papst Franziskus am 25. März 2019, in: http://w2.vatican.va/content/francesco/de/apost_exhortations/d ocuments/papa-francesco_esortazione-ap_20190325_christus-vivit.html (abgerufen am 06.04.2020); Botschaft von Papst Franziskus zum Weltgebetstag für die Bewahrung der Schöpfung am 1. September 2019, in: http://www.vatican.va/conten t/francesco/de/messages/pont-messages/2019/documents/papa-francesco_20190901 _messaggio-giornata-cura-creato.html (abgerufen am 06.04.2020); Nachsynodales apostolisches Schreiben Querida Amazonia von Papst Franziskus am 2. Februar 2020, in: http://www.vatican.va/content/francesco/de/apost_exhortations/docume nts/papa-francesco_esortazione-ap_20200202_querida-amazonia.html (abgerufen am 06.04.2020). 
Medard Kehl qualifiziert die Option für die Armen als einen bedeutsamen Perspektivenwechsel:

„Die Armen können nicht mehr als ,Objekte“ einer paternalistisch sich $\mathrm{zu}$ ihnen herablassenden Kirche behandelt werden. In einer Kirche mit den Armen, die sich in die Welt der Armen hineinbegibt und deren Bedingungen freundschaftlich-solidarisch teilt, werden die Armen selbst zu tragenden Subjekten der Kirche und ihres gemeinsamen Glaubens“ (Medard Kehl ${ }^{3} 1994,244-245$ ).

Den Seligpreisungen des Matthäus-Evangeliums folgend, sind die Armen somit nicht mehr nur die moral patients, also Empfänger, sondern die moral agents, diejenigen, die damit tätig werden. Prominente Vertreter"innen der Option für die Armen sprechen in Hinblick auf das versteckte, strukturell bedingte Leiden statt von den Armen vom "gekreuzigten Volk“, das im Schweigen leidet (Ignacio Ellacuria 1996). Diese theologische Interpretation soll im vorliegenden Beitrag auf ihre potentielle Tragfähigkeit für den Bereich des nicht-menschlichen Lebens hin untersucht werden. Bewusst werden Fragen der Hierarchisierung des Lebens - zum Beispiel bezüglich der Intelligenz, des Schmerzempfindens oder der Ähnlichkeit an sich - ausgeklammert. Wichtig nun an der oben vorgestellten Interpretation ist die Tatsache, dass Armut keineswegs (mehr) ausschließlich an materiellen Mitteln festgemacht wird. Diesen Umstand haben sich bereits andere Teilbereiche, insbesondere mithilfe Martha Nussbaums Capabilities-Ansatzes (Martha Nussbaum 1999 und 2010), nutzbar gemacht. ${ }^{12}$

Unter Anbetracht der Tatsache, dass Papst Franziskus darauf verweist, dass, wenn „das Herz wirklich offen ist für eine universale Gemeinschaft [...], nichts und niemand aus dieser Geschwisterlichkeit ausgeschlossen“ (LS 92) werden könne, müssen auch Tiere in ihrem Leiden beachtet werden. Hierfür wird vorausgesetzt, dass sich die hier thematisierten Tiere menschbedingt langfristig in einer Situation befinden, die es ihnen nicht erlaubt, ihren Bedürfnissen nachzukommen und ihre „Wünsche“ zu realisieren.

12 Im deutschsprachigen theologischen Raum sei an dieser Stelle auf den Schulbereich (Anna Noweck 2013 und 2014), Menschen- und Frauenrechte (Cornelia Mügge 2017) und Modernisierungsverlierer*innen (Roland Mierzwa 2016) als moderne Anwendungsbereiche verwiesen. 


\subsection{Zwischen Paternalismus und Anwaltschaft}

Der Spagat, den die Option für die Armen heute in all seiner Perfektion beherrschen muss, wird zwischen Paternalismus und Anwaltschaft geschlagen. Gemeinhin bezeichnet ersterer eine (übergebührliche) Bevormundung, bei letzterem dagegen wird mehr auf eine Kooperation auf Augenhöhe geachtet. Dabei steht das politische Schlagwort des „aktivierenden Sozialstaats" häufig im Zentrum der Diskussion. Dass dieser Graben aber auch in andere Bereiche, namentlich die hier angedachte Inklusion nichtmenschlichen Lebens in das Konzept der Option für die Armen hinein weist, zeigt, dass es sich nicht nur um ein Problem aus dem Feld der Sozialpolitik handelt, sondern um eine sehr viel fundamentalere Frage, nämlich, wie gut etwas sein kann, das gut gemeint ist.

Denn wenn sich im allgemeinen Sprachgebrauch auch heute der Konsens verbreitet hat, dass Paternalismus abzulehnen sei, gibt es doch verschiedene Bereiche des täglichen Lebens, die sehr wohl nach seinen Regeln gestaltet sind: so stehen neben der klassischen Erziehungsverantwortung von Eltern gegenüber ihren (minderjährigen) Kindern auch staatliche Regelungen, wie beispielsweise die Anschnallpflicht. Als weitergehendes Beispiel wird häufig die strafrechtliche Verfolgung von Menschen, die dem Tötungswunsch einer anderen Person nachgekommen sind, erwähnt.

Wichtig ist diese Infragestellung der oft vorschnellen Kritik an paternalistischen Regelungen, weil über sie die Hauptargumentationslinie bei der Anwendung der Option für die Armen für nicht-menschliche Tiere geht: die institutionell-advokatorische Verantwortungsübernahme und Bevormundung wird gemeinhin auch von Vertreter*innen der Option für die Armen abgelehnt, stellt aber im vorliegenden Fall eine sehr viel gangbarere Möglichkeit dar als die anwaltschaftliche Vertretung, bei welcher der perspektivischen Voraussetzung natürliche Grenzen gesetzt sind. Diese Grenzen können aber bedingt, mit Rückgriff auf Peter Singer (1996), auf Säuglinge oder schwer demente Personen verwischt werden, denen in seinem Konzept weniger „Personsein“ zukommt als ausgewachsenen, gesunden Primaten.

Tom Regan (2003) löst dieses Problem durch die Unterscheidung zwischen moral agents (moralisch Handelnden) und moral patients (moralisch Behandelten). Moral agents sind hierbei diejenigen, von denen eine Handlung oder auch Macht ausgeht. Systembedingt also in diesem Zusammenhang Menschen. Moral patients hingegen sind diejenigen, denen eine Behandlung widerfährt.

Analog zu dem selbstverständlichen Grundsatz, dass Individuen, die weder Moral begreifen, gestalten oder im Umgang mit anderen berücksichti- 
gen können, dennoch einen elementaren Schutz durch diese Regeln erfahren, sei dies auch für Tiere einzufordern (Markus Vogt 2019, 341).

Deshalb sollte in Bezug auf nicht-menschliche Tiere zwar weiterhin beachtet werden, dass Anwaltschaft auch aus der Perspektive der Option für die Armen der vorzuziehende Blickwinkel ist (Katja Winkler 2014), ein tier-theologischer Ansatz aber auch einer gewissen Interpretationsoffenheit bedarf bzw. seine Anwendung die Vorzeichen berücksichtigen muss, unter denen er formuliert wird. Beispielsweise kann und muss die Erziehung eines Tieres, sofern es denn mit Menschen zusammenleben soll, auch respektvoll geschehen. Unzählige Beispiele „sozial nicht verträglicher“ Hunde in Tierheimen machen dies deutlich. Außerdem werden besonders Hunde häufig als Familienmitglieder wahrgenommen, was sie in ihrer Qualität als zu erziehende Teile der Familie in die Nähe der eingangs erwähnten Kinder rückt und somit aus dem Feld der Paternalismus-Kritik befreit.

\subsection{Ausgestaltung in unterschiedliche Kategorien}

Giancarlo Collet (1992) bemühte sich vor allem in den 1990erund 2000er-Jahren als Direktor des Instituts für Missionswissenschaften an der Universität Münster intensiv um die Systematisierung der (vorrangigen) Option für die Armen im deutschsprachigen Bereich. Dabei lag sein Fokus auf der Berücksichtigung der je unterschiedlichen kontextualen $\mathrm{Zu}$ gänge, weshalb er die Rede von den „Christentümern“ prägte (aufgegriffen unter anderem durch Arndt Bünker et al. [Hg.] 2010). Mit diesem Neologismus wollte er dem auch im Christentum noch zu stark verbreiteten Eurozentrismus entgegentreten und der u. a. in der Option für die Armen grundgelegten Orientierung am einzelnen Leidenden Referenz zollen.

Seine Analyse der „Ursprungstexte“ der Option für die Armen ergab, dass letztlich mit den Texten von Medellín (1968), namentlich Nr.14 der Veröffentlichung, ein Rückgriff auf Lumen gentium, Nr. 8, vorliegt, ohne dass dieser deutlich gemacht würde ${ }^{13}$ : die zwingend wahrzunehmende Ar-

13 Giancarlo Collet 1992, 73, verweist hierfür auf: Dokument 14: „Armut der Kirche“, Nr. 7, wo LG 8 jedoch nicht zitiert wird. Der Text findet sich in: Die Kirche Lateinamerikas. Dokumente der II. und III. Generalversammlung des Lateinamerikanischen Episkopates in Medellín und Puebla, hrsg. vom Sekretariat der Deutschen Bischofskonferenz, Bonn ohne Jahr (Stimmen der Weltkirche 8). Zum hermeneutischen Stellenwert vor allem dieses Dokumentes für das Verständnis von Medellín vgl. Josef Amstutz 1989, 174-178. 
mut einzelner Glieder der Kirche wird einmal von oben, Rom, und einmal von unten, arme Ortskirche, aufgegriffen. Das Ergebnis wird durch den pastoraltheologischen Dreischritt Sehen - Urteilen - Handeln in der Reihenfolge Analyse - Theologische Reflexion - Pastorale Leitlinien dargestellt (Giancarlo Collet 1992, 74). Mithilfe dieser Reflexion lassen sich vier unterschiedliche Kernelemente der Option für die Armen deduzieren, oder, anders gewendet, kann die Option für die Armen hierdurch aus vier unterschiedlichen Zugängen heraus begründet werden: theologal, analytisch, politisch und partizipativ (Giancarlo Collet 1992; Andreas Fisch 2001).

Grundsätzlich bedeutet theologal in diesem Zusammenhang, dass Gott aus sich selbst heraus die Armen erwählt und in seine besondere Gnade aufgenommen hat. Analytisch hat eine doppelte Stoßrichtung: einerseits soll die Gesellschaft allgemein auf ihr armmachendes Konfliktpotential hin untersucht werden, andererseits stehen die Christen sowie die Kirche in ihrem Verhalten gegenüber Armen im Zentrum der Betrachtung. Im Fokus der politischen Option steht ganz klar, die Gründe der Armut zu bekämpfen. Dazu gehört beispielsweise die Veränderung der der Armut zuträglichen Faktoren, aber auch die Kooperation mit unterschiedlichen (Laien-)Organisationen mit einem ähnlichen Ziel. Der partizipative Aspekt nun bezieht sich auf die subjekthafte Annahme des Anderen unter gleichzeitiger Akzeptanz seines Andersseins. Ergänzend kommt noch hinzu, dass die Betroffenen sich erneut ihres Glaubens auch gegenüber den sie Unterdrückenden ermächtigen sollen. Dies umfasst sowohl die Deutung der Glaubensinhalte als auch die Verkündigung des Wortes Gottes aus der unterdrückten Position heraus: Verkündende"r und Hörende*r müssen sich hierfür als Teile der unterdrückten Gesellschaftsschicht verstehen.

Auf den hier vertretenen Ansatz, die Option für die Armen auf ihre mögliche Übertragbarkeit auf nicht-menschliche Arme zu untersuchen, ergibt sich für die vier dargestellten Aspekte folgendes Bild: theologal kann argumentiert werden, dass genauso wie Gott menschliche Arme erwählt und in seiner besonderen Gnade aufgenommen hat, dies auch mit nichtmenschlichen Armen der Fall ist. Neben klassischen Beispielen aus dem Alten und dem Neuen Testament (Ex 1-15; Ps 34,7; Lk 4,18-19; Mt 25,35-36) kann und muss an dieser Stelle natürlich auf den franziskanischen Sonnengesang verwiesen werden. Die Analyse der Situation und heutigen Beachtung von nicht-menschlichen Tieren - es sei nur auf die eingangs erwähnten Interviewabschnitte verwiesen - lässt darauf hoffen und auch realistisch schließen, dass ein Umdenken zumindest ansatzweise stattfindet. Auch gesamtgesellschaftlich lassen die aktuelle Diskussion um vegane Ernährung und das leider nicht sehr weit gediehene Tierwohllabel 
gleichwohl darauf schließen, dass sogar die einseitige Sicht von Nutztieren als Lebensmittellieferanten langsam zumindest in gewissen Milieus überholt ist. Politisch gäbe es ein breites Feld möglicher Ansatzpunkte, nichtmenschliche Tiere in ganz unterschiedlichen Situationen, die aus verschiedensten Gründen unter Menschen leiden, zu schützen, zu stärken und zu respektieren.

Im Gegensatz zu den anderen drei Kernelementen der Option für die Armen scheint die Umsetzung der Forderung bzw. die Anwendung der Partizipation für und auf Tiere am schwierigsten. So kann die Idee der Akzeptanz des Anderen in seiner Andersartigkeit letztlich auch auf nichtmenschliche Tiere übertragen werden. An der Forderung jedoch, nichtmenschliche Tiere sich selbst wieder ihres Glaubens bemächtigen zu lassen, scheiden sich die Geister. Biblisch könnte auf Bileams Eselin, die Gottes Präsenz sehr viel früher als er selbst spürt/sieht, verwiesen werden (Num 22,21-34). Gregor Taxacher $(2018,66)$ verweist auf verschiedene Stellen der Legenda aurea, in denen Tieren ein „Sensorium“ für das Göttliche und Übernatürliche zugeschrieben wird. Es könnte aber auch dafür plädiert werden, dass es bereits zwischen Menschen schwierig ist, den Grad des Glaubens des anderen nachzuvollziehen und unterschiedliche kulturelle Kontexte diese Einordnung (oder Beurteilung?) noch weiter verzerren. Wieviel schwerer ist dann eine objektive Beurteilung der Glaubensfähigkeit von Tieren? Da die letzte Forderung jedoch ein Recht darstellt, könnte in Bezug auf nicht-menschliche Tiere argumentiert werden, dass ein Recht nicht ausgeübt werden muss, um Gültigkeit zu haben. Deshalb sollte es potentiell zumindest nicht vorschnell abgesprochen werden.

Die skizzierte Anwendung der vier Bereiche auf nicht-menschliche Tiere zeigt Grenzen und Möglichkeiten auf, wo und wie die Ausweitung der Forderungen der Option für die Armen auch für nicht-menschliche Arme fruchtbar gemacht werden könnte. Dabei muss die Forderung Collets stärker berücksichtigt werden, die Kontextualität der Option für die Armen mitzudenken. Es gilt, den einzelnen Armen, sei er nun menschlicher oder nicht-menschlicher Natur, zu sehen und wahrzunehmen mit allem, was er kann, und auch mit all dem, was er nicht kann.

\section{Rufnach christlicher Tierethik und theologischer Beachtung von Tieren muss beantwortet werden}

Die im Verlauf des Artikels vorgebrachten Argumente und Verbindungen zeigen, wie wichtig und auch im Sinne der Option für die Armen dringend nötig eine weitere Reflexion nicht-menschlichen Lebens vor dem ak- 
tuellen Hintergrund ist. Dass sie bereits stattfindet, zeigen die Interviewergebnisse zu Beginn des Beitrags nachdrücklich. Warum diese weiterverfolgt werden muss, ergibt sich aus der sich aus naturwissenschaftlichen Studien und Untersuchungen her- und abzuleitenden Erkenntnis, dass die Trennung zwischen Tier und Mensch nicht existiert. Wie jedoch diese Begründung auch einerseits theologisch verortet werden kann und dadurch eine für manche Menschen vielleicht noch weitergehende Schlagkraft entfalten kann, sollte durch die Zusammenführung der Aufsprengung des immer noch dominanten Anthropozentrismus in der katholischen Kirche und der Christenheit im allgemeinen als auch der Wahrnehmung des Tieres an sich als ein unter ,arm“ zu subsummierenden Wesens erreicht werden.

Dass es unterschiedliche Stufen von Schmerzempfinden, Bewusstsein, Intelligenz und Kommunikation innerhalb und zwischen unterschiedlichen Tierarten gibt, kann in diesem Zusammenhang außer Acht gelassen werden, denn letztlich geht es dem hier vorgestellten Ansatz darum, das Tier als Gegenüber wahrzunehmen und damit in seinem Sein und eben nicht nur in seinem Nutzen wahrzunehmen (Julia Blanc 2011). Dieser Ansatz folgt der vertieften modernen Auseinandersetzung mit dem menschlichen Gegenüber: initial durch Johannes XXIII. geprägt, wird die Option für die Armen nachdrücklich besonders in Lateinamerika - Medellín $(1968)^{14}$ und Puebla (1979) ${ }^{15}$ müssen als die wohl wichtigsten Versammlungen in diesem Zusammenhang genannt werden - geprägt. Durch das Werk von Papst Franziskus wurde die anthropozentrische Fixierung, wie sie vor allem im Zuge des 2. Vatikanischen Konzils etabliert wurde, in Teilen aufgeweicht. Die erneute Betonung der Mitgeschöpflichkeit - hierbei vor allem aus dem Wissen indigener Völker und deren Umgang mit der Schöpfung abgeleitet - sollte es ermöglichen, klare Abgrenzungen zwischen dem Menschen als „Krone der Schöpfung“ und dem Rest der Schöpfung als ihm „dienende Ergänzung“, wie es die Forschung aus unterschiedlichsten Bereichen nahelegt, zu überwinden. Dies sollte es auch dem theo-

14 Obwohl der Ausdruck „Option für die Armen“ hier noch keine namentliche Erwähnung fand, wurde das Konzept durch das dortige Treffen der lateinamerikanischen Bischofskonferenz geprägt. Die Abschlussdokumente wurden vom Lateinamerikanischen Bischofsrat (CELAM) 1968 veröffentlicht.

15 Der Lateinamerikanische Bischofsrat spricht 1979 diesbezüglich von der „vorrangigen Option für die Armen“. 
logischen Diskurs ermöglichen, die Idee der Grenzen zugunsten von Übergängen und Abstufungen weiterzuentwickeln. ${ }^{16}$

\section{Literatur}

Amery, Carl 1972, Das Ende der Vorsehung. Die gnadenlosen Folgen des Christentums, Reinbek.

Amstutz, Josef 1989, Medellín: Die eine Absicht in den vielen Beschlüssen, in: Neue Zeitschrift für Missionswissenschaft 45, 174-197.

Aseneta, Anatoly Angelo R. 2017, Laudato Si' on Non-Human Animals, in: Journal of Moral Theology 6 (2), 230-245.

Barbato, Mariano Pasquale 2018, Keep the Faith: Progress, Social Justice and the Papacy, in: Smith, Peter J./Glaab, Katharina/Baumgart-Ochse, Claudia/Smythe, Elizabeth (Hg.) 2018, The Role of Religion in Struggles for Global Justice. Faith in justice?, Abingdon, 1157-1171.

Blanc, Julia 2011, Nachhaltige Entwicklung - für wen?, in: Demele, Markus/Hartlieb, Michael/Noweck, Anna (Hg.) 2011, Ethik der Entwicklung. Sozialethische Perspektiven in Theorie und Praxis, Münster, 73-79.

Blanc, Julia 2017, Ökokatholizismus. Sozialethische Analysen zu ausgewählten Ländern und Institutionen in Europa, Marburg.

Bünker, Arndt/Mundanjohl, Eva/Weckel, Ludger/Suermann, Thomas (Hg.) 2010, Gerechtigkeit und Pfingsten. Viele Christentümer und die Aufgabe der Missionswissenschaft, Ostfildern.

Camosy, Charles C. 2015, „Laudato Si’ on Non-Human Animals: Three Hopeful Signs, Three Missed Opportunities" (25.06.2015), in: https://catholicmoraltheology.com/laudato-si-on-non-human-animals-three-hopeful-signs-three-missed-opportunities/ (abgerufen am 05.05.2020).

Camosy, Charles C. 2016, „Locating Laudato Si' along a Catholic Trajectory of Concern for Non-Human Animals“, in: Lex Naturalis 2, 1-20.

Camosy, Charles/Kopp, Susan 2014, „The Use of Non-Human Animals in Biomedical Research: Can Moral Theology Fill the Gap?“, in: Journal of Moral Theology 3 (2), 54-71.

CELAM 1968, 2a Conferencia General del Episcopado Latinoamericano (Puebla), Bogota.

16 Ich möchte mich für die Möglichkeit bedanken, dass ich diese Gedanken erstmals beim 39. Fachkongress der internationalen Vereinigung für Moraltheologie und Sozialethik in Brixen vorstellen durfte. Mein besonderer Dank gilt Martin M. Lintner mit den anderen Organisatoren für die Annahme des Papers, Kerstin Schlögl-Flierl für die Moderation und Markus Vogt und Sigrid Müller für ihre Nachfragen und Denkanstöße. 
CELAM 1979, 3a Conferencia General del Episcopado Latinoamericano (Medellín), Bogota.

Collet, Giancarlo 1992, Europa in der Verantwortung gegenüber Lateinamerika 500 Jahre nach der Eroberung des Kontinents, in: Jahrbuch für Christliche Sozialwissenschaften 34, 67-84.

Deutsche Bischofskonferenz (Hg.) 1979, Die Kirche Lateinamerikas. Dokumente der II. und III. Generalversammlung des Lateinamerikanischen Episkopates in Medellín und Puebla (Stimmen der Weltkirche 8), Bonn.

Dimaghani, Maryam 2018, Which is greener: secularity or religiosity? Environmental philanthropy along religiosity spectrum, in: Environmental Economics and Policy Studies 20 (2), 477-502.

Ellacuria, Ignacio 1996, Das gekreuzigte Volk, in: Ellacuria, Ignacio/Sobrino, Jon (Hg.) 1996, Mysterium Liberationis. Grundbegriffe der Theologie der Befreiung 2, Luzern, 823-850.

Fisch, Andreas 2001, Option für die Armen konkret: zur sozialethischen Kompetenz der Kirche in Deutschland (Forum Religion und Sozialkultur, Abt. B Profile und Projekte 4), Münster.

Franziskus 2013, Evangelii gaudium. Apostolisches Schreiben über die Verkündigung des Evangeliums in der Welt von heute (24.11.2013).

Franziskus 2015, Laudato si'. Enzyklika über die Sorge für das gemeinsame Haus (24.05.2015).

Glaab, Katharina/Fuchs, Doris/Friedrich, Johannes 2019, Religious NGOs at the UNFCCC. A specific contribution to global climate politics?, in: BaumgartOchse, Claudia/Wolf, Klaus-Dieter (Hg.) 2019, Religious NGOs at the United Nations. Polarizers or Mediators, Abingdon, 47-63.

Grim, John 2011, The roles of religions in activating an ecological consciousness, in: International Social Science Journal 62, 255-269.

Gutiérrez, Gustavo ${ }^{10} 1992$, Theologie der Befreiung. Mit der neuen Einleitung des Autors und einem Vorwort von Johann Baptist Metz, Mainz.

Häring, Bernard 1981, Free and Faithful in Christ: Moral Theology for Priests and Laity, vol. 3, Light to the World, Salt of the Earth, New York.

Horstmann, Simone 2020, Was fehlt, wenn uns die Tiere fehlen? Eine theologische Spurensuche, Regensburg.

Horstmann, Simone/Ruster, Thomas/Taxacher, Gregor 2018, Alles, was atmet: Eine Theologie der Tiere, Regensburg.

Johannes Paul II. 1979, Redemptor hominis. Enzyklika über den Erlöser des Menschen (04.03.1979).

Johannes Paul II. 1987, Solicitudo rei socialis. Enzyklika über die Sorge über die sozialen Anliegen (30.12.1987).

Johannes Paul II. 1990, Friede mit Gott, dem Schöpfer - Friede mit der ganzen Schöpfung. Botschaft zum Weltfriedenstag (01.09.1990).

Johannes Paul II. 1991, Centesimus annus. Enzyklika zum hundertsten Jahrestag von Rerum novarum (01.05.1991). 
Kehl, Medard ${ }^{3}$ 1994, Die Kirche. Eine katholische Ekklesiologie, Würzburg.

Leo XIII. 1891, Rerum novarum. Enzyklika über die Arbeiterfrage (15.05.1891).

Lintner, Martin M. 2017, Der Mensch und das liebe Vieh. Ethische Fragen im Umgang mit Tieren. Mit Beiträgen von Christoph J. Amor und Markus Moling, Innsbruck.

Lintner, Martin M. 2020, Respect for the Proper Value of each Creature. An Animal-ethical Rethinking of the Encyclical Laudato si', in: Louvain studies 42 (1), 26-48.

Linzey, Andrew 2009 a, Creatures of the Same God: Explorations in Animal Theology, New York.

Linzey, Andrew 2009 b, Why Animal Suffering Matters: Philosophy, Theology, and Practical Ethics, Oxford.

Maibach, Edward/Leiserowitz, Anthony/Roser-Renouf, Connie/Myers, Theresa/ Rosenthal, Seth/Feinberg, Geoff 2015, The Francis effect: How Pope Francis changed the conversation about global warming, Fairfax, VA; online: https:// climatecommunication.yale.edu/wp-content/uploads/2015/11/The_Francis_Effect.pdf (abgerufen am 29.01.2021).

Meadows, Donella/Meadows, Dennis/Randers, Jørgen/Behrens, William 1972, The limits to growth. A report for the Club of Rome's project on the predicament of mankind, New York.

Mierzwa, Roland 2016, „Option für die Armen“ angesichts der Globalisierung: Der Konsultationsprozess der deutschen Kirchen zu wirtschaftlichen und sozialen Fragen (1994-1997), Norderstedt.

Mügge, Cornelia 2017, Menschenrechte, Geschlecht, Religion. Das Problem der Universalität und der Fähigkeitenansatz von Martha Nussbaum, Bielefeld.

Münk, Hans J. 1987, Umweltkrise - Folge und Erbe des Christentums? Historischsystematische Überlegungen zu einer umstrittenen These im Vorfeld ökologischer Ethik, in: Jahrbuch für Christliche Sozialwissenschaften 28, 133-206.

Noweck, Anna 2013, Katholische Schulen - beteiligungsgerecht? Eine sozialethische Untersuchung unter besonderer Berücksichtigung des Capabilities Approach, Bielefeld.

Noweck, Anna 2014, Schule mit den Armen. Die Option für die Armen als Kriterium gerechter Gestaltung von Bildung, in: Blanc, Julia/Brinkschmidt, Maria/ Krauß, Christoph/Reichert, Wolf-Gero (Hg.) 2014, Armgemacht - ausgebeutet ausgegrenzt? Die „Option für die Armen“ und ihre Bedeutung für die Christliche Sozialethik (Forum Sozialethik 14), Münster, 107-126.

Nussbaum, Martha 1999, Gerechtigkeit oder Das gute Leben, herausgegeben von Herlinde Pauer-Studer, Frankfurt a. M.

Nussbaum, Martha 2010, Grenzen der Gerechtigkeit. Behinderung, Nationalität, Spezieszugehörigkeit, Berlin. 
Ostheimer, Jochen 2018, Der Wert des Tieres und sein Nutzen für den Menschen. Tierethische Reflexionen zur Xenotransplantation, in: Sautermeister, Jochen (Hg.) 2018, Tierische Organe in menschlichen Körpern. Biomedizinische, kulturwissenschaftliche, theologische und ethische Zugänge zur Xenotransplantation, Paderborn, 429-449.

Palmer, Martin 2013, The quiet revolutionaries: why the faith-based environmental movement has become the largest civil society movement in the conservation world, in: Biodiversity 14 (3), Abingdon, 180-182.

Paul VI. 1967, Populorum progressio. Enzyklika über den Fortschritt der Völker (26.03.1967).

Rappel, Simone 1996, „Macht euch die Erde untertan“. Die ökologische Krise als Folge des Christentums, Paderborn.

Regan, Tom 2003, Animal Rights, Human Wrongs: An Introduction to Moral Philosophy, Lanham/USA.

Remele, Kurt 2016, Die Würde des Tieres ist unantastbar. Eine neue christliche Tierethik, Kevelaer.

Rosenberger, Michael 2015, Der Traum vom Frieden zwischen Mensch und Tier. Eine christliche Tierethik, München.

Schockenhoff, Eberhard ${ }^{2} 2013$, Ethik des Lebens. Grundlagen und neue Herausforderungen, Freiburg i. Br. etc.

Schwantes, Milton 2002, Es fließe das Recht der Armen. Die Armen und die Bibel, in: Ordensnachrichten 41 (5), Wien, 3-10.

Singer, Peter 1996 [1975], Animal Liberation. Die Befreiung der Tiere, Reinbek.

Taxacher, Gregor 2018, Den Drachen töten und die Spatzen füttern. Die Heiligung der Natur in der Legenda Aurea, in: Horstmann, Simone/Ruster, Thomas/Taxacher, Gregor 2018, Alles, was atmet. Eine Theologie der Tiere, Regensburg, 63-78.

Taylor, Bron 2006, The Encyclopedia of Religion and Nature, London/New York City.

Tucker, Mary Evelyn/Grim, John (series editors) 1997-2004, Religions of the World and Ecology Book Series, London.

Tutu, Desmond 2013, Extending Justice and Compassion. Foreword, in: Linzey, Andrew (Hg.) 2013, The Global Guide to Animal Protection, Urbana etc., xv.

Vogt, Markus 2013, Prinzip Nachhaltigkeit. Ein Entwurf aus theologisch-ethischer Perspektive, München.

Vogt, Markus 2018, Die Zehn Gebote des Bodenschutzes. Schöpfungsethische Leitlinien zum Umgang mit Land, in: Stimmen der Zeit 143, 265-275.

Vogt, Markus 2019, Tierethik. Philosophische und theologische Ansätze des gegenwärtigen „animal turn“, in: Münchener Theologische Zeitschrift 70, 333-354.

White, Lynn 1967, The Historical Roots of Our Ecologic Crisis, in: Science, New Series 155, No. 3767, 1203-1207. 
Winkler, Katja 2014, Armut und Paternalismus, in: Blanc, Julia/Brinkschmidt, Maria/Krauß, Christoph/Reichert, Wolf-Gero (Hg.) 2014, Armgemacht - ausgebeutet - ausgegrenzt? Die „Option für die Armen“ und ihre Bedeutung für die Christliche Sozialethik (Forum Sozialethik 14), Münster, 89-106.

Wustmans, Clemens 2015, Tierethik als Ethik des Artenschutzes. Chancen und Grenzen (Ethik - Grundlagen und Handlungsfelder 9), Stuttgart. 
